\title{
Scanning Electron Microscopy of Corrosion Cast of Rat Adrenal Vasculatures with Emphasis on Medullary Artery under ACTH Administration
}

\author{
Katsuhiro Nakamura and Takayuki Masuda* \\ Department of Clinical Research, Sendai National Hospital, \\ Sendai 980 and *the First Department of Pathology, Akita \\ University School of Medicine, Akita 010
}

\begin{abstract}
Nakamura, K. and Masuda, T. Scanning Electron Microscopy of Corrosion Cast of Rat Adrenal Vasculatures with Emphasis on Medullary Artery under ACTH Administration. Tohoku J. exp. Med., 1981, 134 (2), 203-213In the vascular system of the adrenal, the medullary artery is considered to be a shunt which controls cortical function by changing its diameter. To confirm this hypothesis, the medullary arteries of ACTH-treated and control rats were observed by scanning electron microscopy of methacrylate corrosion casts of blood vessels. After ACTH treatment, the medullary arteries were constricted mostly at their distal segments and sometimes also at other portions. This constriction probably results in an effective augmentation of adrenocortical blood flow. The diameter of capillaries in the glomerular zone and medullary sinusoids was markedly increased by ACTH. Small protrusions on the corrosion casts which supposedly corresponded to capillary fenestrations became prominent after ACTH administration in the fascicular and reticular zones. - scanning electron microscopy; corrosion cast; rat adrenal blood vessel
\end{abstract}

The vascular system of the mammalian adrenal gland is rather complicated because of the coexistence of two embryologically different tissues, the cortex and medulla. As for the arterial system, a stem artery gives off two kinds of arteries on the capsule of the adrenal. One is the cortical artery which empties into capillaries of the glomerular zone and the other is the medullary artery which passes through the cortex without giving off any branch to the cortex and empties into medullary sinusoids (Harrison and Hoey 1960).

Meanwhile, in the adrenal medulla, phenylethanolamine $\mathrm{N}$-methyl transpherase (PNMT) which converts norepinephrine to epinephrine by transmethylation is known to be activated by a large amount of corticosteroid (Pophorecky and Wurtman 1971). Therefore, norepinephrine producing cells show a tendency to assemble around an opening of the medullary artery where the concentration of corticosteroid is supposed to be diluted (Weiss and Greep 1977).

However, the distribution pattern of medullary arteries differs largely from species to species of animals according to the macroscopical configuration of the adrenal. For instance, the rat adrenal has a spherical configuration and concentric 
vascularization and is supplied with one to four medullary arteries, whereas the human adrenal shows a plate-like configuration and has hundreds of medullary arteries (Harrison 1960). Therefore, regional differences in the portal and arterial circulations are expected to be markedly accentuated in the rat adrenal. In accordance with the difference in vascularization, the tendency of assemblance of norepinephrine cells around an opening of the medullary artery is remarkable in rats, while it is indistinct in man. Moreover, it is difficult in the human adrenal even to distinguish norepinephrine cells from epinephrine cells (Nakamura 1977).

Another probable effect of the medullary artery on the function of the adrenal gland is expected from the fact that the medullary artery is a shunt vessel for the adrenal cortex. The administration of ACTH to a dog has been known to increase adrenal blood flow (Urquhart 1965). Under this condition, if the blood flow through the medullary artery remains unchanged, the blood flow through the adrenal cortex must increase effectively. In this respect, the authors intended to observe a change in diameter of the rat medullary artery after ACTH treatment. Methyl methacrylate corrosion casts of the adrenal blood vessels were prepared and observed with a scanning electron microscope.

\section{Materials and Methods}

Twenty adult male Wistar strain rats were divided into control and ACTH-treated groups. Ten animals were injected intramuscularly with 10 units of ACTH once a day for 7 days. They were sacrificed $1 \mathrm{hr}$ after the last injection.

The abdominal and chest walls of the animals of both groups were opened under Nembutal $^{\circledR}$ anesthesia. Fifty $\mathrm{ml}$ of physiological saline solution to which was added a few drops of heparin was perfused into every animal from the left ventricle of the heart, cutting off the vena cava at the same time. Immediately after the perfusion of saline, another needle was inserted into the descending aorta and $100 \mathrm{ml}$ of a mixture of $5 \%$ paraformaldehyde and $5 \%$ glutaraldehyde solution adjusted to $\mathrm{pH} 7.2$ with $0.1 \mathrm{M}$ cacodylate buffer was injected within approximately $5 \mathrm{~min}$. Then, $50 \mathrm{ml}$ of methacrylate resin (Mercox) was injected within 2 to $3 \mathrm{~min}$. After polymerization of resin the aorta, kidneys and adrenals were removed en bloc from the animals.

These organs were immersed in $20 \% \mathrm{KOH}$ solution to remove tissues from the resin casts. It took 1 to 2 weeks at room temperature. KOH solution was changed every 2 to 3 days. The resin casts of the adrenal blood vessels were split carefully with forceps and a razor blade under a dissecting microscope. Then, they were coated with gold using an ion sputter (JFC-1100, JEOL). The observation was made with a scanning electron microscope (T-20, JEOL) at magnifications $\times 35$ to $\times 5000$.

\section{Results}

\section{Control group}

Cortical arteries. In the rat, usually three stem arteries entered into each of the adrenal glands. They were various in diameter ranging from 60 to $90 \mu \mathrm{m}$. However, as a sequence of arborization in the periadrenal tissue, the diameters of the three stem arteries became almost equal just prior to the first bifurcation on the adrenal capsule. Each stem artery repeated dichotomous branching three to six times on the adrenal capsule prior to emptying into capillaries (Fig. 1). The mean 
diameters of them are shown in Fig. 1. As for the left adrenal, one or sometimes two stem arteries were originated from the left renal artery (Fig. 2). On the surface of the resin casts, a long series of circular constrictions perpendicular to the long axis of the artery was observed. It occurred most frequently on the cast of a blood vessel with a diameter of less than $20 \mu \mathrm{m}$, that is they were corresponding to

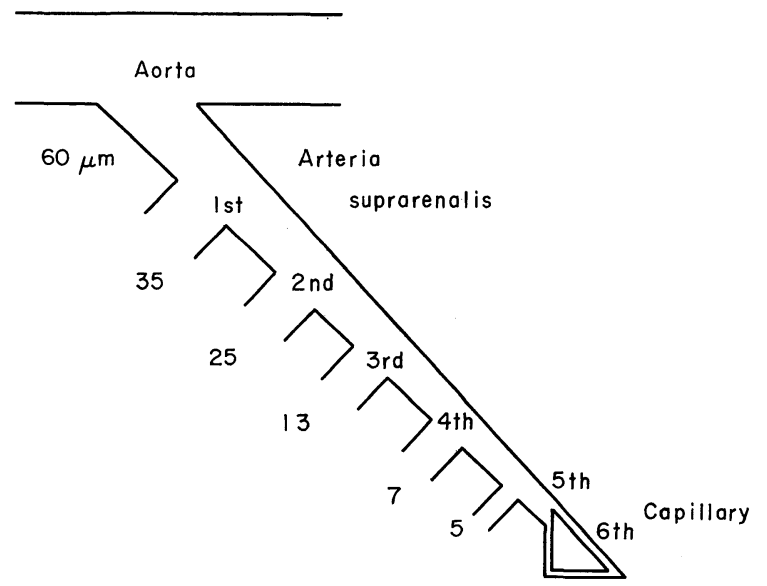

Fig. 1. Scheme showing mean diameters of each subbranch of the arteria suprarenalis of control rats.

cortical arteries formed by the third or fourth bifurcation of the stem artery. The intervals of circular constrictions were 3 to $5 \mu \mathrm{m}$.

Medullary arteries. The medullary artery which arose from the stem artery passed through the glomerular zone sometimes in spiral fashion, then ran straight downwards without giving off any branches, and finally emptied into medullary sinusoids or capillaries in the reticular zone (Fig. 3). The mean diameter of the medullary arteries was $25 \mu \mathrm{m}$. This value corresponded to that of the second dichotomous branch. In this experiment, however, we could not acertain in every case how many numbers of dichotomous branchings of the stem artery yielded to the medullary artery, because the continuation of the cast from a stem artery to the medullary artery was usually destructed in the process of specimen preparation. For instance, there was a cortical artery which was a counterpart of the medullary artery showing four times dichotomous branching before it emptied into the capillary plexus; the diameter of this medullary artery was $10 \mu \mathrm{m}$. This value seems lower than that of the third dichotomous branching.

It should be noticed that there was little difference in diameter between the proximal portion of the medullary artery and its distal portion just proximal to the medullary sinusoid (Table 1).

Instead of circular constrictions observed in cortical arteries, deep streaks other than nuclear impressions of the endothelial cells were observed along the 


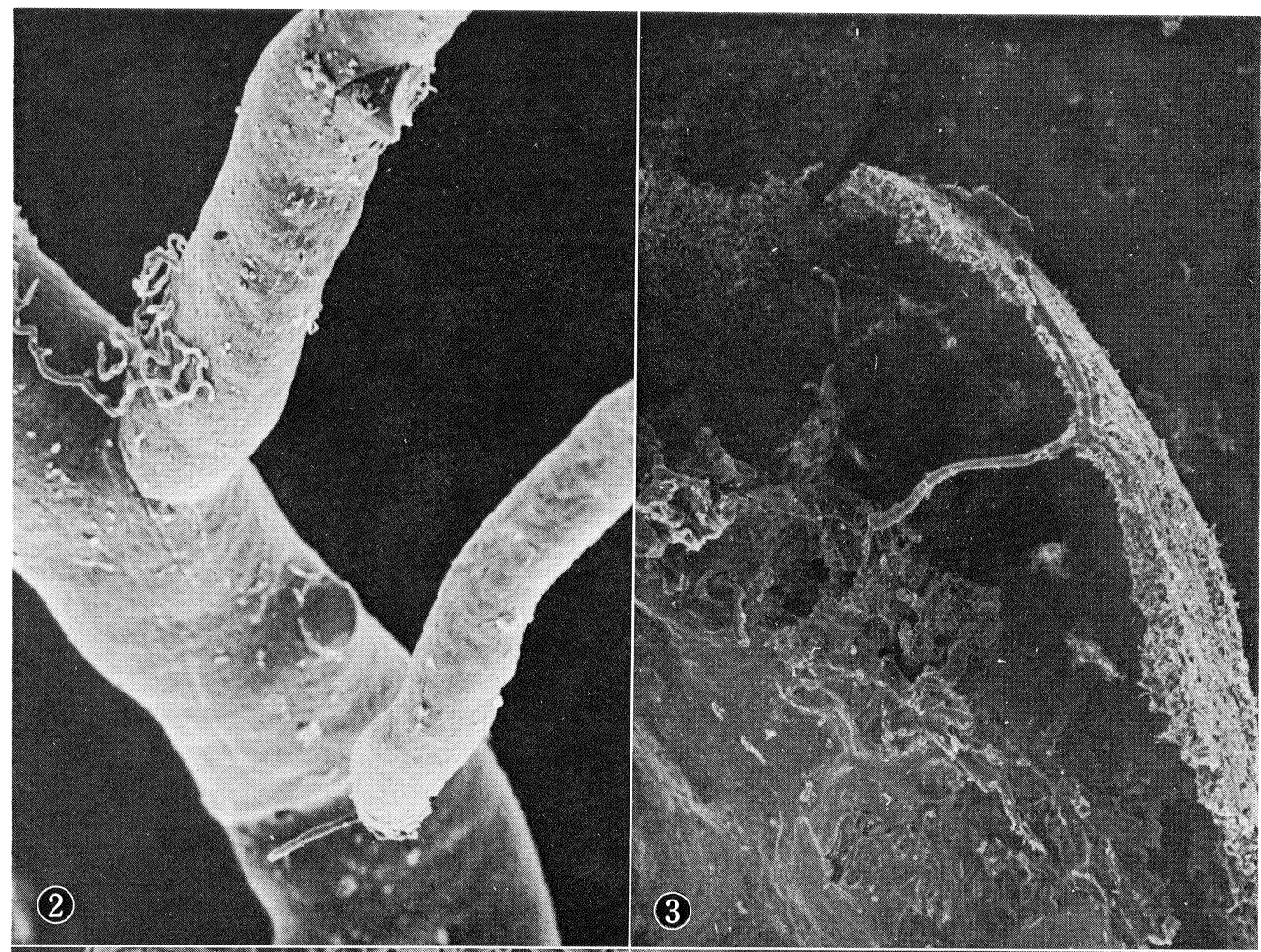

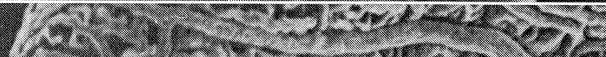

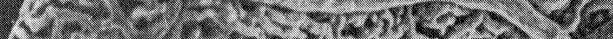

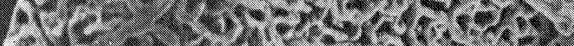

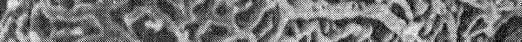

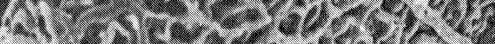
in 12.

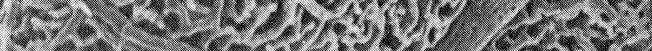

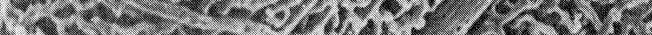
Lo s =

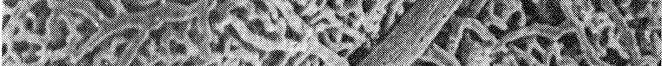

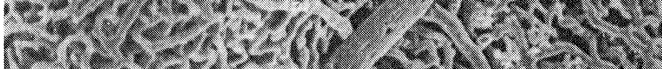

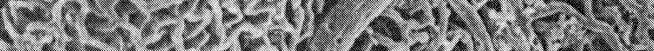

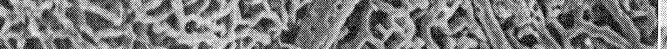

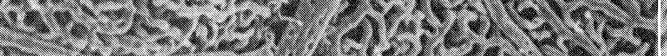

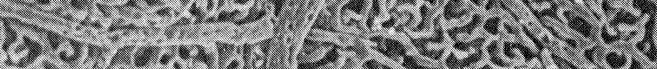
s.

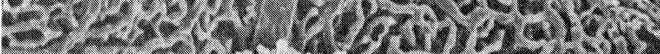
\% 4.

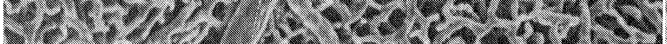

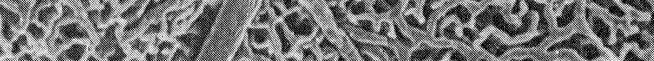
3 s.

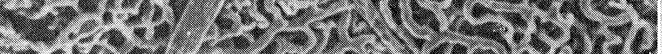

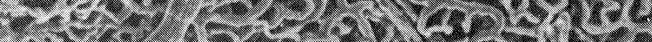

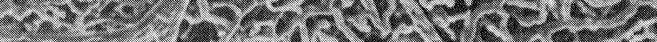

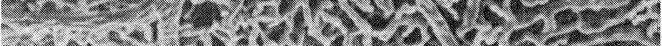

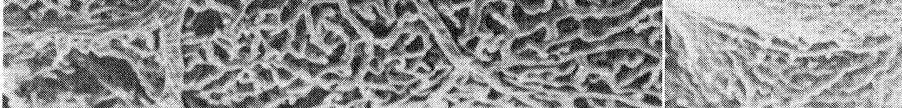

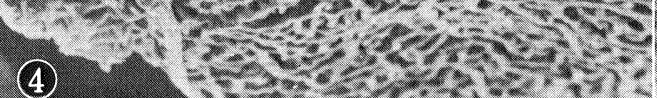
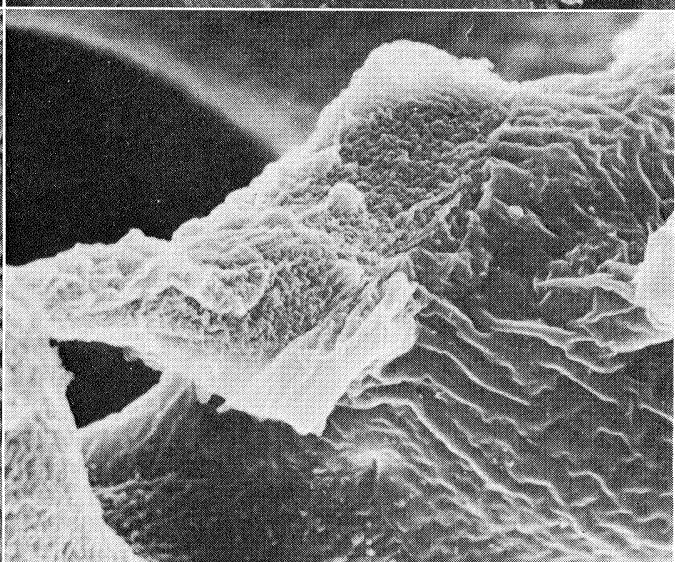
ind 0 (w)

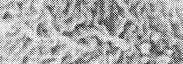

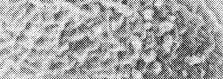
$7 x^{2}=0-25$ $52^{2}=$ 
TABLE 1. Difference in diameter between proximal and distal portions of the medullary artery

\begin{tabular}{cccccc}
\hline \multicolumn{2}{c}{ Control } & & \multicolumn{2}{c}{ ACTH } \\
\cline { 2 - 3 } \cline { 5 - 5 } Proximal & Distal & & Proximal & Distal \\
\hline 40 & 30 & & 50 & 20 \\
30 & 40 & & 20 & 20 \\
30 & 30 & & 25 & 15 \\
25 & 20 & & 20 & 5 \\
20 & 25 & & 35 & 15 \\
10 & 20 & & & \\
Mean \pm s.D. & $25 \pm 9.75$ & $24.3 \pm 10.97$ & $30 \pm 12.74$ & $15 \pm 6.12$ \\
\hline
\end{tabular}

Values are expressed as $\mu \mathrm{m}$.

long axis of the medullary artery. They were more frequent in the distal portion of the vessel. Small spherical protrusions, 0.5 to $1 \mu \mathrm{m}$ in diameter, on the surface of the cast of medullary arteries were sometimes observed, but they were rather a few in number.

Capillaries. The capillaries anastomosed each other to make a lattice-like structure beneath the capsule. The size of each lattice was approximately $15 \times$ $15 \mu \mathrm{m}$ (Fig. 4). Then, the capillaries immediately changed the direction perpendicularly to the capsule. Therefore, as far as vascular architecture was concerned, the glomerular zone was not distinct in the rat. The authors hereafter refer to the capillaries located just beneath the capsule as those of the glomerular zone. The mean diameter of capillaries of every zone increased toward the distal portion (Table 2). Meanwhile in the reticular zone, anastomoses between capillaries gradually increased in number. In the medulla, capillaries joined each other to make balloon-like sinusoids and reduced their number remarkably.

Sometimes narrow junctions were observed between the capillaries of the reticular zone and the medulla.

Under higher magnifications a small number of spherical protrusions, 0.5 to $1 \mu \mathrm{m}$ in diameter, on the surface of corrosion casts of the glomerular zone were observed. Towards the fascicular and reticular zones, these protrusions increased in number gradually. Sometimes, there occurred oval protrusions, $1 \times 2 \mu \mathrm{m}$, other than the spherical ones. In the sinusoid of the medulla spherical protrusions again decreased remarkably in number. Sometimes on the casts of capillaries of every zone a wrinkling structure was observed, but it was not always a constant finding (Fig. 5).

Fig. 2. The cortical artery and capillary network beneath the adrenal capsule. Nuclear impressions are observed on the cortical artery. Control. $\times 1,000$.

Fig. 3. Two left adrenal stem arteries branching from the left renal artery. Control. $\times 200$.

Fig. 4. The medullary artery connecting the adrenal capsule and the medulla. Control. $\times 75$.

Fig. 5. Wrinkling structures of capillaries of the reticular zone. Control. $\times 20,000$. 


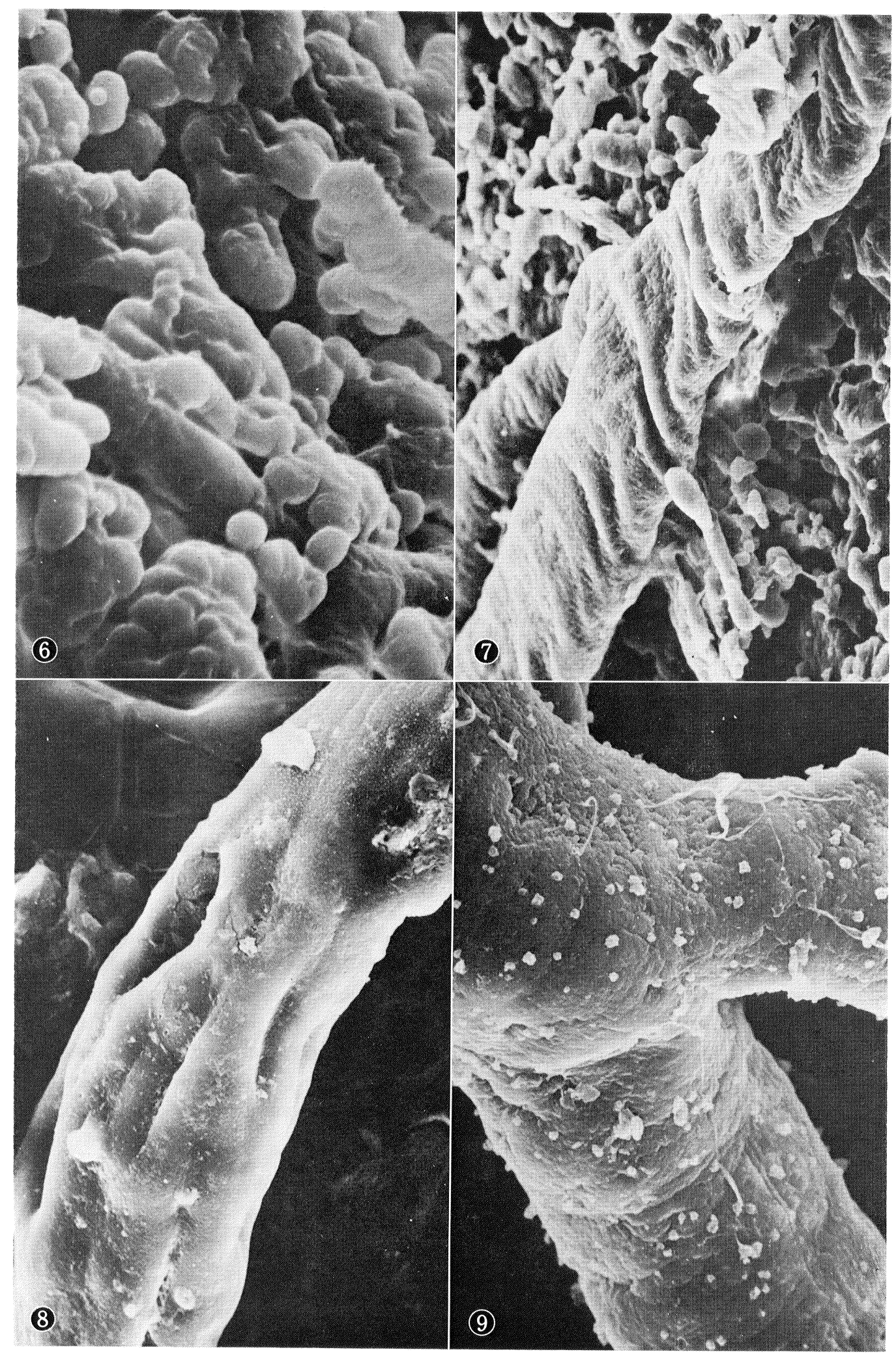


Though fine granular protrusions were sometimes observed on the surface of the casts obtained from capillaries of the medulla, it was difficult to confirm that they corresponded to capillary fenestrations because they were not always constant findings. In the glomerular zone, a group of small spherical extravascular configurations, sized 1 to $2 \mu \mathrm{m}$, were observed (Fig. 6). They were sometimes connected with

TABLE 2. The diameter of capillaries of each zone of the adrenal

\begin{tabular}{lrr} 
& \multicolumn{1}{c}{ Control } & \multicolumn{1}{c}{ ACTH } \\
\hline Z. glomerulosa & $4.01 \pm 1.27$ & $5.00 \pm 1.78$ \\
Z. fasciculata & $5.45 \pm 1.78$ & $8.10 \pm 2.71$ \\
Z. reticularis & $10.59 \pm 2.82$ & $11.09 \pm 2.73$ \\
Medulla & $22.65 \pm 9.68$ & $36.23 \pm 14.87$ \\
\hline
\end{tabular}

Values are expressed as mean \pm s.D. $(\mu \mathrm{m})$.

capillaries as if they leaked from the vessels. However, it was not certain whether they were merely leakage from the capillary or they represented lymph vessels. Nuclear impressions were generally observed on the casts from every sort of vessel, though they appeared less frequently on the capillary than on the artery (Fig. 4).

\section{$A C T H$-treated group}

Cortical arteries. The cortical arteries increased in diameter correspondingly to the enlargement of the adrenal induced by ACTH administration. The mean diameter of the cortical arteries ranged from 90 to $120 \mu \mathrm{m}$.

The circular constrictions perpendicular to the long axis of the vessels became distinct (Fig. 7).

Medullary arteries. The size of medullary arteries was generally larger than that of the control group. The difference in diameter between proximal and distal portions of the vessel was marked. Usually the diameter of the distal portion was remarkably smaller than that of the proximal portion (Table 2). In accordance with narrowing of the vessel, the deep concave structures along the long axis of the vessel became conspicuous (Fig. 8). In a few cases, however, narrowing of the medullary arteries was observed also in proximal portions of the vessels.

Capillaries. The diameter of capillaries in the glomerular zone was increased

Fig. 6. Small spherical extracapillary configurations. Control. $\times 4,000$.

Fig. 7. Remarkably developed precapillary sphincters of the cortical artery observed in an ACTH-treated rat. Small spherical extracapillary configurations are prominent. $\times 1,000$.

Fig. 8. A distal portion of the medullary artery of an ACTH-treated rat. Indentations along the long axis of the vessel is conspicuous. $\times 2,000$.

Fig. 9. Capillaries of the reticular zone of an ACTH-treated rat. Spherical and/or ovoid protrusions become more prominent both in number and in size than those of the control animal. $\times 2,000$. 
by more than $20 \%$ as compared with that of the control group (Table 2). Small spherical protrusions, 0.5 to $1 \mu \mathrm{m}$, on the capillary of the glomerular zone were a féw as in the control group, while the spherical extravascular configurations, 1 to $2 \mu \mathrm{m}$, became more remarkable both in number and in size.

In the fascicular and reticular zones the diameter of the capillaries increased only slightly. The small spherical protrusions increased both in number and in size. Moreover, oval or rod-shaped ones also increased (Fig. 9).

In the medulla, the sinusoids again dilated approximately twice in size as compared with those of the control group. Small spherical protrusions on the casts were less numerous than in the control group.

\section{Discussion}

With administration of a large amount of ACTH, the rat adrenal gland increased in dimension approximately twice as large as that of the control animals. At the same time, the three stem arteries, as well as the cortical arteries, increased in diameter approximately twice the control. It has been known that the arterial blood flow is in proportion to $r^{2.7}$ ( $r$ : radius of the artery) (Suwa and Takahashi 1971). While, in the ACTH-administered group, the medullary artery on one side dilated twice the size of the control, the medullary artery on the other side was equal to or even smaller than in the control.

As seen in Table 3, if the diameter of an artery which gives off the medullary artery by dichotomy increases $A$ times that in the control and the diameters of its two branches are equal, then blood flow through each branch increases to $A^{2.7} \times V$ ( $V$ : original blood flow through each brach). However, under the same condition, if the diameter of the medullary artery, one of the two branches, remains unchanged, the blood flow through the two branches is $V$ and $\left(2 A^{2.7}-1\right) \cdot V$, respectively. Therefore, an increase of blood flow through the cortical artery is augmented by $\left(\left(2 A^{2.7}\right.\right.$ $\left.\left.1) \cdot V-A^{2.7} \cdot \mathrm{V}\right)\right) / 2 A^{2.7} \cdot V \times 100=50\left(1-1 / A^{2.7}\right)(\%)$ owing to the stenotic effect of the medullary artery. Namely, if the diameter of the stem artery which gives off two branches, medullary and cortical arteries, increases twice, the stenosis of the medullary artery further adds about $40 \%$ of blood flow through the stem artery to the cortical artery.

This augmentation of cortical blood flow is expected to occur only in the portion where one of the two branches is the medullary artery. Therefore, it is necessary to estimate how much the ratio of this increase is to the total adrenal blood flow. As seen in Fig. 1 and Table 2, the average diameter of the medullary arteries was approximately corresponding to that of the cortical arteries which were given off by the second bifurcation of the stem artery. However, there was one medullary artery whose diameter was smaller than the average and nearly corresponding to the diameter of the third bifurcation of the stem artery. This medullary artery was considered to be yielded by the second bifurcation of the stem artery as judged from the number of dichotomous branchings until it emptied into the capillary. Thus, the medullary arteries seem to be given off mostly after 


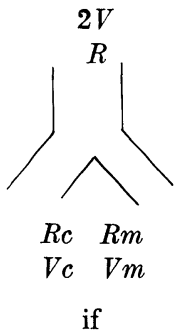

$R c=R m$ $V c=V m=V \quad$ and

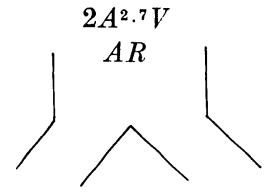

$R c^{\prime} \quad R m^{\prime}$

$V c^{\prime} \quad V m^{\prime}$

if

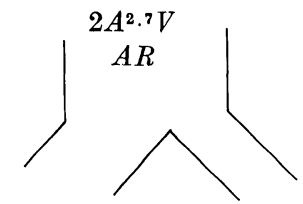

$R c^{\prime \prime} R m$

$V c^{\prime \prime} \quad V m$

Augmented increase (\%):

$\frac{\left(2 A^{2.7}-1\right) V-A^{2.7} V}{2 A^{2.7} V} \times 100=50\left(1-1 / A^{2.7}\right)$

$R:$ Radius of the stem artery.

$R c, R c^{\prime}, R c^{\prime \prime}$ : Radius of the cortical artery.

$R m, R m^{\prime}, R m^{\prime \prime}$ : Radius of the medullary artery.

$2 V:$ Volume of blood flow through stem artery.

$V c, V c^{\prime}, V c^{\prime \prime}$ : Volume of blood flow through cortical artery.

$V m, V m^{\prime} V m^{\prime \prime}$ : Volume of blood flow through medullary artery.

$A$ : Constant.

the second bifurcation. But we could not rule out the possibility that the third bifurcation of the stem artery yields the medullary artery.

Here, we propose a model of the adrenal which has two medullary arteries; one is given off from a stem artery by the second bifurcation and the other is given off by the third bifurcation.

Further, we assume that an arterial branch is divided dichotomously into two subbranches of an equal diameter and that the diameters of three stem arteries are also equal. Blood flows through the second and third subbranches are $F / 3 \times$ $1 / 4$ and $F / 3 \times 1 / 8$, respectively ( $F$, total adrenal blood flow). When the total adrenal blood flow increases twice and the medullary arteries keep their original diameters unchanged, the percentage of increase in blood flow through the cortical arteries which are the counterparts of the medullary arteries is $25 \%$ (cf. Table 3 , in this case $A^{2.7}=2$ ). Therefore, the ratio of this value to the total adrenal blood flow is $(1 / 12+1 / 24) F \times 0.25 \fallingdotseq 0.03 F$. In the actual adrenal of the rat, this value is expected to become somewhat higher because of the following reasons: 1) The number of medullary arteries in one adrenal of the rat was usually more than 2 . 2) The narrowest part of the medullary artery after administration of ACTH was even narrower than the control. 3) Usually the stem artery of the adrenal gives off some branches to extraadrenal tissues before and after the first bifurcation.

Harrison (1957) in his microradiographical observations mentioned probable constriction of the medullary artery after administration of epinephrine. However, 
he did not directly observe the constriction of medullary artery, but he suggested it from the fact that the capillary filling with thorium dioxide was more remarkable after epinephrine administration. When a large amount of epinephrine is discharged in the adrenal medulla where it acts on the medullary artery to constrict it, there may occur an accelerated discharge and production of corticosteroid which may in turn yield increasing conversion of norepinephrine to epinephrine via PNMT activation (Pophorecky and Wurtman 1971). This hypothesis, therefore, seems attractive from the view point of recovery mechanism from shock, though PNMT activation with corticosteroid is not so prompt but rather a slow reaction. It should be mentioned, however, that Harrison (1957) did not observe capillary filling with thorium dioxide at least within $30 \mathrm{~min}$ after epinephrine administration. That is, he injected thorium dioxide antecedently to the injection of epinephrine. One of the present authors has observed that $3 \mathrm{~min}$ after thorium dioxide injection, a large amount of colloidal particles leaked out from the capillary and that an uptake of the particles by extracapillary mesenchymal cells as well as by parenchymal cells occurred (Nakamura 1973). Therefore, it is possible that the enhancement of capillary filling noted by Harrison (1957) resulted from extracapillary leakage and precipitation of thorium dioxide which were induced by increasing permeability of the capillary. Whether the function of the medullary artery is under the control of some chemicals such as ACTH, epinephrine, angiotensine and so on or simply under the control of blood pressure should be studied further.

Another problem of the constricting mechanism of the medullary artery is that in the present experiments no distinct structures reflecting circular smooth muscles were observed around the medullary artery of the rat. Around the cortical artery a long series of circular constrictions were observed. These configurations correspond to precapillary sphincters (Baez 1977). Around the medullary artery of the rat, long indentations along the long axis were observed instead of circular sphincters. They were quite different in size from nuclear impressions (Anderson and Anderson 1978). They were particularly marked after ACTH administration when the distal portion of the medullary artery was constricted. These facts suggest the existence of a constriction mechanism mostly at the distal portion of the medullary artery.

As for the capillary of the adrenal, the change of its diameter induced by ACTH administration was interesting. By ACTH administration, dilatation of the capillary in the glomerular zone and the medulla was remarkable, but it was not distinct in the fascicular and reticular zones. By ACTH administration spherical or ovoid protrusions which may correspond to the capillary pores were markedly increased in number as well as in size in the reticular and fascicular zones. From these facts, it can be assumed that, in the adrenal cortex part of increased blood flow would leak out into the parenchyme owing to increased permeability of the capillary. On the contrary, the capillary of the medulla which has a few pores seems to adapt to increasing blood flow by dilatation of the lumen.

As for the wrinkling structures which were observed sometimes on the capillaries 
in every zone, it was not distinct whether they represented boundaries of endothelial cells or not. Neither was it clear which structures on the corrosion cast represented fenestrations of the capillary, though we sometimes observed fine protrusions on the cast. To solve these problems further improvements in the methods for specimen preparation will be awaited.

\section{Acknowledgment}

We express sincere thanks to Mr. Shigeki Endo for his technical assistance and to Miss Hiroko Hasebe for her secretary assistance.

\section{References}

1) Anderson, B.G. \& Anderson, W.D. (1978) Scanning electron microscopy of microcorrosion cast: Intracranial and abdominal microvasculature in domestic animals. Amer. J. Anat., 153, 523-536.

2) Baez, S. (1977) Microvascular terminology. In: Microcirculation, vol. 1, edited by G. Kally \& B.M. Altura, University Park Press, Baltimore, Maryland, pp. 23-34.

3) Harrison, R.G. (1957) Adrenal circulation in the rabbit. J. Endocr., 15, 64-71.

4) Harrison, R.G. \& Hoey, M.J. (1960) The Adrenal Circulation. Blockwell Scientific Publication, Oxford, p. 9.

5) Nakamura, K. (1973) An electron microscopic observation on RE-cells of the rat adrenal cortex with respect to the nature of lipid globules. Tohoku J. exp. Med., $109,205-221$.

6) Nakamura, K. (1977) Hormone Producing Tumor. 1st ed., edited by S. Ishikawa et al., Igaku Shoin, Ltd., Tokyo, pp. 148-159. (Japanese)

7) Pophorecky, L.A. \& Wurtman, R.J. (1971) Adrenocortical control of epinephrine synthesis. Pharmacol. Rev., 23, 1-35.

8) Suwa, N. \& Takahashi, T. (1971) Morphological and Morphometrical Analysis of Circulation in Hypertension and Ischemic Kidney, Urban \& Schwarzenberg, MünchenBerlin-Wien, pp . 10-22.

9) Urquhart, J. (1965) Adrenal blood flow and the adrenocortical response to corticotropin. Amer. J. Physiol., 209, 1162-1168.

10) Weiss, L. \& Greep, R.O. (1977) Histology. 4th ed., Mcgraw-Hill Book Co., New York, p. 1113. 